\title{
Belief AHP method: AHP method with the belief function framework
}

\author{
Amel Ennaceur, Zied Elouedi, Eric Lefevre
}

\begin{abstract}
In this paper, the Analytic Hierarchy Process (AHP) method is extended to an uncertain environment where the uncertainty is represented by belief functions as interpreted in the Transferable Belief Model (TBM). Our proposed approach, called belief AHP, is developed to help the decision maker to determine what the best alternatives are, considering multiple conflicting criteria where both alternatives and criteria may be soiled with imperfection. The Belief AHP method aims at comparing subsets of criteria and groups of alternatives in order to reduce the pair-wise comparisons number. Furthermore, to handle uncertainty that may appear in the comparison procedure, we use basic belief assignments instead of exact ratios to elicitate expert preferences. Finally, to illustrate the feasibility of our approach and to judge its performances, we have applied our proposed method on a real application problem: we have considered the PVC life cycle assessment especially the end of life phase.
\end{abstract}

Keywords - Multi-criteria decision making; AHP; Belief function theory; Belief pair-wise comparison; Uncertain preferences.

\section{Introduction}

In real life decision making situation, the decision maker faces with many cases in which he has to make a decision among different alternatives. However, the most preferable one is not always easily selected. So, the expert often needs to make judgments on alternatives that are evaluated on the basis of different criteria. [7] [9] Within this framework, many methods have been proposed and each one has its own characteristics. In the following, we will focus on the Analytic Hierarchy Process (AHP), [1] [10] [11] since it is amongst one of the most well known methods.[30] The strength of this approach is that it is easier to understand and it can effectively handle both 
qualitative and quantitative data.[34] [35] However, the AHP method is often criticized [2] [3] [31] due to the exponentially increase of the number of pair-wise comparisons. In reality, the elicitation of preferences may be rather difficult when the number of alternatives and criteria is large. If the number of alternatives (criteria) in the hierarchy increases then, more comparisons are needed to be made. Another weakness of the AHP is its inability to handle the inherent uncertainty of the pair-wise comparison process. Typically, the scale is bounded and it is a verbal description of the intensity of preference and the Saaty scale is used, although any other scale could be used in this method.[27] In some cases, expert would not be able to efficiently express any kind of preference degree between the available alternatives and criteria. This problem of judgment inconsistency has also be handled by some existing works. [42] [43]

In order to overcome these difficulties and to extend the AHP on a more realistic elicitation procedure, several AHP methods are combined within uncertain theories. One of these extensions is the Fuzzy AHP, [13] which utilized triangular fuzzy numbers to model the pair-wise comparisons. Since then, several fuzzy AHP developments have been proposed.[14] Besides, probabilistic AHP methods are introduced in,[15] handling pairwise comparison matrices based on probability theory, where each element of which is the prior probability. There are also other ways to solve AHP problems.[16] [32] [36] [37]

In particular in the belief function framework, Beynon et al. have proposed a method called the DS/AHP method [17] [18] comparing not only single alternatives, but also groups of alternatives. Besides, several works have been proposed by Utkin.[26] [29] The main feature of his approach is that it allows the expert to deal with comparisons of arbitrary subsets of alternatives and criteria.[33] Additionally, Smarandache et al. [19] have developed the DSmT/AHP which is based on the Dezert-Smarandache theory.[20] This method aimed at performing a similar purpose as DS/AHP that is to compare groups of alternatives. All these extensions are very useful and important. However, it requires introducing additional assumptions concerning the corresponding membership functions. Moreover, most elicitation procedures in this case are based on standard pair-wise comparisons which have some limits. Therefore, our proposed solution avoids the standard pair-wise comparisons and proposes a new elicitation technique based on the belief function framework. The expert has then the ability to express his assessment freely, because in some cases, he is unable to model his preferences due to absence of knowledge, lack experience, etc. The objective is then to extend the approach presented in [6] under a new comparison technique. In 
[6], we construct groups of alternatives and criteria, but we use the Saaty scale to compare each element of the hierarchy.

In this paper, we present what we call a belief AHP approach, a multicriteria decision making (MCDM) method adapted to imprecise and incomplete preferences, where the uncertainty is represented by belief functions as defined in the Transferable Belief Model (TBM). The choice of the TBM seems appropriate as it provides a convenient framework for dealing with incomplete and uncertain information, notably those given by experts. This theory is chosen because it has a powerful evidence combination rule, and it represents properly partial and total ignorance; it assigns beliefs to individual elements of the hypothesis set as well as their subsets. Our aim through this work is to propose an uncertain AHP approach. On one hand, our suggested solution offers a formalism for reducing the number of pairwise comparisons. It will be able to compare groups of alternatives instead of comparing only single alternatives between each other. To judge the importance of criteria, our approach offers tools allowing the expert to express his ranking even over subgroups of criteria.[6] Our method is then able to compare groups of criteria at the criterion level and groups of alternatives at the alternative level. Moreover, we are interested in treating the uncertainty that may appear in the comparison procedure. Hence, our solution moves from a scaled level of preference to response based on "yes" or "no". Then, the expert responses are described by a distributed assessment using a belief structure. The main objective of introducing uncertainty in the comparison scale is to model both precise data and subjective judgments with uncertainty can be consistently modeled under this framework. Rather than forcing the expert to provide exact representations of imprecise perceptions, we suggest using an imprecise representation instead. Also, using the belief function framework, we have the possibility to express our total ignorance, which was impossible under standard AHP.

Finally, to illustrate the feasibility of our approach, we have applied our proposed method on a real application problem: we have considered the PVC life cycle especially the end of life phase (the disposal problem). The challenge facing an expert here is the choice of the country where the environmental impact is the least important for the destruction of a kilogram of PVC.

In what follows, we first present an overview of the basic concepts of respectively the AHP and the belief function theory. Then, in the main body of the paper, we present our new approach: the belief AHP which is based on the belief function theory. Finally, our method will be illustrated by a real application problem in order to understand its real unfolding and 
to show the applicability of the developed approach.

\section{Basic concepts}

In this section, we briefly introduce a number of the key concepts in order to better understand our contribution.

\subsection{Analytic Hierarchy Process}

The AHP approach is a decision-making technique developed by Saaty [10] [11] to solve complex problems of choice and prioritization. The problem is structured hierarchically at different levels. The purpose of constructing this hierarchy is to evaluate the influence of the criteria on the alternatives to attain objectives. So, an AHP hierarchy has at least three levels: The highest level consists of a unique element that is the overall objective. Then, each level of the hierarchy contains criteria or sub-criteria that influence the decision. Alternative elements are put at the lowest level.

Once the hierarchy is built, the decision maker starts the prioritization procedure to determine the relative importance of the elements on each level of the hierarchy. Elements of each level are paired (with respect to their upper level decision elements) and then compared. This method elicits preferences through pair-wise comparisons based on a nine-point scale,[10] which translates the preferences of a decision maker into crisp numbers (see Table 1).

Table 1: The Saaty rating scale

\begin{tabular}{c|c}
\hline Saaty's scale & Definition \\
\hline 1 & Equal importance \\
3 & Somewhat more important \\
5 & Much more important \\
7 & Very much more important \\
9 & Absolutely more important \\
$2,4,6,8$ & Intermediate values \\
\hline
\end{tabular}

After filling all the pair-wise comparison matrices, the local priority weights are determined by using the eigenvalue method. The objective is then to find the weight of each criterion, or the score of each alternative by calculating the eigenvalue vector. With these values, the AHP method permits to compute a consistency ratio to check if the matrix is consistent 
or not. The last step aggregates all local priorities from the decision table by a simple weighted sum. The global priorities thus obtained are used for final ranking of the alternatives and the selection of the best one.

\subsection{Belief function theory}

In this subsection, we briefly review the main concepts underlying the belief function theory as interpreted by the TBM. Details can be found in [21] [22].

\subsubsection{Basic concepts}

Let $\Theta$ be the frame of discernment representing a finite set of elementary hypotheses related to a problem domain. We denote by $2^{\Theta}$ the set of all the subsets of $\Theta$.

The impact of a piece of evidence on the different subsets of the frame of discernment $\Theta$ is represented by the so-called basic belief assignment (bba), called initially basic probability assignment. [4] It quantifies the impact of a piece of evidence on the different subsets of the frame of discernment.[22]

A bba is a function denoted by $m$ that assigns a value in $[0,1]$ to every subset $A$ of $\Theta$ such that:

$$
\sum_{A \subseteq \Theta} m(A)=1
$$

A basic belief mass (bbm) $m(A)$ quantifies the portion of belief that supports $A$ without supporting any strict subset of $A$, and that could be transferred to subsets of $A$ if further information justifies it.

\subsubsection{Combination}

The belief function theory offers interesting tools for combining beliefs induced by distinct pieces of evidence. Let $m_{1}$ and $m_{2}$ be two bba's defined on the same frame of discernment and are issued from distinct sources. One of the combination rules is the conjunctive rule: [21]

$$
\left(m_{1} @ m_{2}\right)(A)=\sum_{B, C \subseteq \Theta, B \cap C=A} m_{1}(B) m_{2}(C)
$$

\subsubsection{Discounting}

The technique of discounting allows us to take in consideration the reliability of the information source that generates the bba $m$. Let $\beta=1-\alpha$ be the degree of reliability $(\alpha \in[0,1])$ assigned to a particular belief function. If 
the source is not fully reliable, the bba it generates is "discounted" into a new less informative bba denoted $m^{\alpha}$ : [24]

$$
\begin{gathered}
m^{\alpha}(A)=(1-\alpha) m(A), \forall A \subset \Theta \\
m^{\alpha}(\Theta)=\alpha+(1-\alpha) m(\Theta)
\end{gathered}
$$

\subsubsection{Coarsening and refinement}

Sometimes, beliefs are induced by information sources with different but compatible frames of discernment. The coarsening and refinement operations allow us to establish relationships between these different frames in order to express beliefs on anyone of them. Let $\Omega$ and $\Theta$ be two finite sets, the idea behind the refinement consists in obtaining one frame of discernment $\Omega$ from the set $\Theta$ by splitting some or all of its events. [4] Conversely, the coarsening consists in forming a frame $\Theta$ by grouping together the events of the frame of discernment $\Omega$ (see Fig. 1).

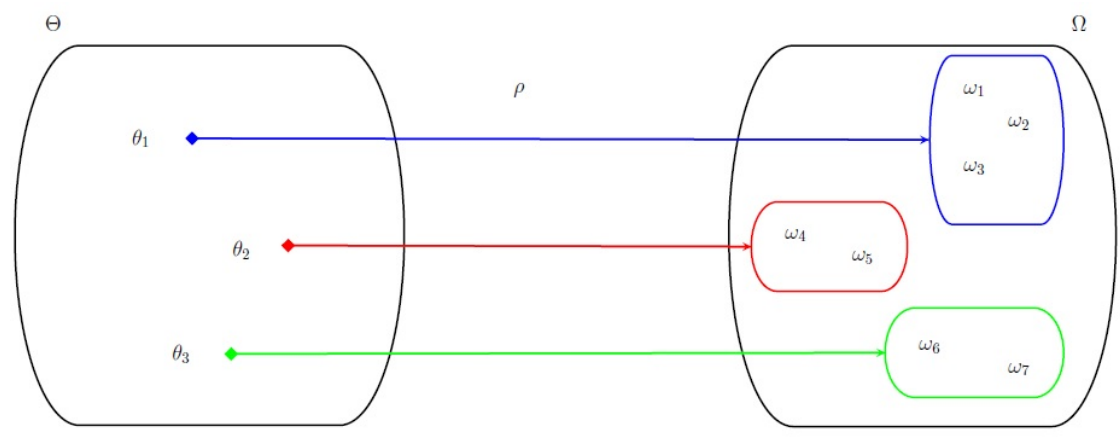

Figure 1: Illustration of a coarsening $\Theta$ of $\Omega$ associated with a refining $\rho$ of $\Theta$.

Let us define a mapping $\rho: 2^{\Theta} \rightarrow 2^{\Omega}$ such that: [4]

$$
\begin{gathered}
\rho(\{\theta\}) \neq \emptyset \quad \forall \theta \in \Theta \\
\rho(\{\theta\}) \cap \rho\left(\left\{\theta^{\prime}\right\}\right)=\emptyset \text { if } \theta \neq \theta^{\prime} \\
\bigcup_{\theta \in \Theta} \rho(\{\theta\})=\Omega
\end{gathered}
$$

So, given a disjoint partition $\rho(\{\theta\})$ one may set: [4]

$$
\rho(A)=\bigcup_{\theta \in A} \rho(\{\theta\})
$$


With this notion of refining, we can define the vacuous extension. This operation allows one to convey a mass function $m^{\Theta}$, expressing a state of belief on $\Theta$, to a finer frame $\Omega$, a refinement of $\Theta$. Stemming from the least committed principle, this operation is denoted with an arrow pointing up, and is defined by:

$$
m^{\Theta \uparrow \Omega}(\rho(A))=m^{\Theta}(A) \quad \forall A \subseteq \Theta
$$

\subsubsection{Decision making}

The TBM considers that holding beliefs and making decision are two distinct processes. Hence, it proposes a two level model:

- The credal level where beliefs are entertained and represented by belief functions.

- The pignistic level where beliefs are used to make decisions and represented by probability functions called the pignistic probabilities, denoted BetP: [5]

$$
\operatorname{BetP}(A)=\sum_{B \subseteq \Theta} \frac{|A \cap B|}{|B|} \frac{m(B)}{(1-m(\emptyset))}, \forall A \in \Theta
$$

\section{Belief AHP approach}

This section is dedicated to the presentation of the basic steps needed to ensure the ranking of alternatives in an uncertain environment. In this context, we introduce our belief AHP approach which is a combination of AHP method and the belief function theory.

\subsection{Identification of the candidate criteria}

Our set of criteria is given by $\Omega=\left\{c_{1}, \ldots, c_{m}\right\}$ where $\Omega$ is the frame of discernment involving all the possible criteria related to the MCDM problem. Denote the set of all subsets of criteria by $2^{\Omega}$, and let $C_{k}$ be a subset of $\Omega$. To identify the candidate criteria, our main idea is to allow the expert to express his opinions on groups of criteria instead of single one. The decision maker chooses these subsets by assuming that criteria having the same degree of preference are grouped together. If an expert identifies a group of criteria, then we could suppose that all of its elements have the same importance. So, by comparing two subsets of criteria $C_{k}$ and $C_{j}$, an expert makes a choice 
between $C_{k}$ and $C_{j}$. Since we are not performing pair-wise comparisons of criteria but relating to groups of criteria, these sets of criteria should not consider a criterion in common, because if one criterion is included in two groups, then each group will give a different level of favorability. By generalization, these subsets can be defined as:

$$
C_{k} \text { is preferred to } C_{j}, \forall k, j \mid C_{k}, C_{j} \in 2^{\Omega}, C_{k} \cap C_{j}=\emptyset
$$

\subsection{Identification of the candidate alternatives}

In many complex problems, experts are able to compare only subsets of alternatives and cannot evaluate separate ones. To solve this problem, that means to reduce the number of alternatives which consequently decreases the number of comparisons, our method suggests not necessarily to consider all of them but just to choose groups of those alternatives. One of the possible solutions of this task is to use the DS/AHP method.[17] [18]

Like the criterion level, we assume that there is a set of all the possible alternatives $\Theta=\left\{a_{1}, \ldots, a_{n}\right\}$ consisting of $n$ elements. Denote the set of all subsets of $\Theta$ by $2^{\Theta}$, and let $A_{k}$ be a subset. At this level, we apply the same hypotheses assumed in DS/AHP to identify the subsets of alternatives. Besides, the decision maker expresses his preferences by comparing groups of alternatives (except the set $\Theta$ which represents the total ignorance on alternatives). For example, $A_{k}$ is preferred to $A_{j}$, that means that $A_{k}$ is more preferable than $A_{j}$. The main aim behind this hypothesis was explained in. [17] [18]

\subsection{Computing the weight of considered criteria}

After identifying the candidate sets of criteria, what is left is "how to calculate the weight of these criteria?". At this point, pair-wise comparisons process is extended to an uncertain environment. We consider the same basic assumptions of AHP method, that all the elements in the same hierarchy are totally independent. In fact, preferences expressed on one criterion do not influence the ones expressed on another criterion.

Under this approach, our proposed scale may be different from the traditional one. In this study, we assume a new set of choices. Thus to express his preferences, the decision maker responses to the following question: "Is the subset of criteria $C_{j}$ important?". Besides, he only selects the related linguistic variable. He indicated whether a criterion was more or less important to its partner by "yes" or "no". In other terms:

$$
\Omega_{C_{j}}=\{y e s, n o\}
$$


where $\Omega_{C_{j}}$ is the set of possible choices.

After identifying the frame of discernment $\Omega_{C_{j}}$ corresponding to each subset of criteria, $C_{j}$, our approach suggests to use a belief pair-wise comparison matrix. In order to express his opinions, we accept that the expert may define uncertain or even unknown assessments. Indeed, we assume that each subset of criteria is described by a basic belief assignment $\left(m^{\Omega_{C_{j}}}\right)$ defined on the possible responses. In other words, to quantify the subjective judgments with uncertainty, a preference degree may be assigned to each decision maker's response. For instance, in a problem of purchasing a car, the following type of uncertain subjective judgments was frequently used: "the comfort criterion is evaluated to be more important than style with a confidence degree of 0.8 ". In fact, the decision maker responses to the question "is comfort criterion important regarding the style criterion?". Thus, the answer was: comfort criterion is more preferable than style criterion and 0.8 is referred to the degree of belief. Finally, the same process is repeated for each pair of subsets.

In some cases, the expert is not able to give any information about alternatives and/or criteria. Thus, the obtained bba will be a vacuous basic belief assignment, and we will get a single subset $m_{j}^{\Omega_{C_{i}}}\left(\Omega_{C_{i}}\right)=1$. This case is referred to as total ignorance. Therefore, the decision maker can express his preferences more freely and all the elicitated data can be modeled.

To model the pair-wise comparison matrix, some priorities must be respected. We consider $X$, the pair-wise comparison matrix, is an $k \times k$ matrix in which $k$ is the number of groups of criteria being compared. It has the following characteristics:

1. The first step is to model the pair-wise comparison matrix. Let $d_{i j}$ is the entry from the $i^{t h}$ column of pair-wise comparison matrix $\left(d_{i j}\right.$ represents the different bbm's of the identified bba).

$$
\bar{m}_{i}^{\Omega_{C_{j}}}(a)=m_{j}^{\Omega_{C_{i}}}(\bar{a})=d_{i j}, \forall a \subset \Omega_{C_{i}}
$$

where $m_{j}^{\Omega_{C_{i}}}$ represents the importance of $C_{i}$ with respect to the subset of criteria $C_{j}$ (for simplicity we denote the subset of criteria by $j$ instead of $\left.C_{j}\right), i \neq j$, and $\bar{m}_{i}^{\Omega_{C_{j}}}$ is the negation of $m_{j}^{\Omega_{C_{i}}}$.

As regarding the previous example, if we have "the comfort criterion $(C)$ is evaluated to be more important than style criterion $(S)$ with a confidence degree of $0.8 "$, that is $m_{S}^{\Omega_{C}}(\{y e s\})=0.8$, then we can say 
that "the style criterion is evaluated to be less important than comfort criterion with a confidence degree of 0.8 ": $m_{C}^{\Omega_{S}}(\{n o\})=0.8$.

2. Like the traditional AHP method, where the principal diagonal contains entries of 1 , we set:

$$
m_{i}^{\Omega_{C_{i}}}\left(\Omega_{C_{i}}\right)=1
$$

Once the pair-wise comparison matrix is complete, our objective is then to obtain the priority of each subset of criteria. In fact, within the belief comparison matrix, our problem is "what is the appropriate function to use in order to obtain a single representation value of these different bbas to get the priority vector". The idea is to combine the obtained bba using the conjunctive rule of combination. Indeed, this function is chosen since we can regard each subset of criteria as a distinct source of information which provides distinct pieces of evidence.

To better understand, we consider $X$, as defined above, the pair-wise comparison matrix. For each row of the matrix, we apply the conjunctive rule. That means, for each subset of criteria $(i=\{1, \ldots, k\})$, we will get the following bba:

$$
m^{\Omega_{C_{i}}}=\bigcirc_{j=1}^{k} m_{j}^{\Omega_{C_{i}}}
$$

Finally, these obtained bba $m^{\Omega_{C_{i}}}$ is transformed into pignistic probabilities, denoted by $\operatorname{Bet}^{\Omega_{C_{i}}}$ using Equation 10 .

The proposed solution addresses the problem of inconsistency. In fact, if the preferences are inconsistent, then we obtain $m(\emptyset)=1$. Thus, the expert may be guided to reformulate his preferences. Otherwise, any belief function will be found.

\subsection{Computing the alternatives priorities}

Following the same reasoning, belief pair-wise comparison matrices are also constructed, and evaluate each group of alternatives regarding each criterion. By applying Equation 15 for each obtained pair-wise comparison matrix, we get a bba which quantifies the degree of belief assigned by the expert to each subset of alternatives. However, our purpose is to combine these obtained bba's to get a single belief function, but the problem here is that each subset of alternatives has its own frame of discernment. For instance, if we say that alternative $A$ is preferred to $B$, and alternative $C$ is preferred to $B$, this does not means that alternative $A$ is indifferent to $C$. Consequently, each obtained bba is defined on a different frame of discernment. 
The idea to use is then to standardize all the frames of discernment. Obviously, we propose to use the concept of refinement operations, [4] which allows us to establish relationships between different frames of discernment in order to express beliefs on anyone of them. The objective is then consists in obtaining one frame of discernment $\Theta$ from the set $\Theta_{A_{k}}$ by splitting some or all of its events. As mentioned above, each bba $m^{\Theta_{A_{k}}}$ represents the belief over all possible answers (yes or no). However, at this stage, we want to know which alternative is the best $(\Theta=\{A, B, C\})$. As a result, $\Theta_{A_{k}}$ is considered as a coarsening of $\Theta$ (see subsection 2.2.4), and we get the following relation:

$$
m^{\Theta_{A_{k}} \uparrow \Theta}\left(\rho_{k}(\omega)\right)=m^{\Theta_{A_{k}}}(\omega) \quad \forall \omega \subseteq \Theta_{A_{k}}
$$

where the mapping $\rho_{k}$ from $\Theta_{A_{k}}$ to $\Theta$ is a refinement, and $\rho_{k}(\{y e s\})=$ $\left\{A_{k}\right\}$ and $\rho_{k}(\{n o\})=\overline{\left\{A_{k}\right\}}$.

Once we have standardized our frame of discernment $\Theta$, we can now combine the resulting bba using the conjunctive rule in order to obtain a belief function reflecting the importance of alternatives to a given criterion:

$$
m_{c_{k}}=\bigcirc_{j=1}^{l} m^{\Theta_{A_{i}} \uparrow \Theta}
$$

where $l$ is the number of subsets of alternatives.

Finally, we obtain $m_{c_{k}}$ representing the opinions-beliefs of the expert about his preferences regarding the set of alternatives.

\subsection{Updating the alternatives priorities}

To update the alternatives priorities with respect to the criterion weight, we have to define a rule for combining them. On the one hand, we have priorities concerning criteria and groups of criteria instead of single ones. On the other hand, the sets of alternatives are compared pair-wise with respect to a specific single criterion.

In order to overcome this difficulty, our approach proposes to regard each pignistic probability distribution of a specific set of criteria as a measure of reliability. This factor is used to measure most heavily the bba evaluated according to the most important criteria and conversely for the less important ones. If we have $C_{k}$ a subset of criteria, then we get $\beta_{k}$ its corresponding measure of reliability:

$$
\beta_{k}=\operatorname{Bet} P^{\Omega_{C_{k}}}(\{y e s\})
$$

As a result, two cases will be presented: First, if the reliability factor represents a single criterion $c_{k}$, then the corresponding bba will be directly discounted: 


$$
\begin{gathered}
m_{c_{k}}^{\alpha_{k}}\left(A_{j}\right)=\beta_{k} \cdot m_{c_{k}}\left(A_{j}\right), \forall A_{j} \subset \Theta \\
m_{c_{k}}^{\alpha_{k}}(\Theta)=\left(1-\beta_{k}\right)+\beta_{k} \cdot m_{c_{k}}(\Theta)
\end{gathered}
$$

where $m_{c_{k}}\left(A_{j}\right)$ the relative bbm for the subset $A_{j}$ (obtained in the previous step: Equation 17), $\beta_{k}$ its corresponding measure of reliability, and we denote $\alpha_{k}=1-\beta_{k}$.

Second, if this factor represents a group of criteria, their corresponding bba's must be combined, then it will be discounted by their corresponding measure of reliability. In this case, each element of a specific group of criteria has its own pair-wise matrix that evaluates the sets of alternatives with respect to this criterion. Therefore, we apply the conjunctive rule of combination to obtain a single representation value of these different bba's. As a result, the obtained bba compares the sets of alternatives according to this set of criteria.

Let $C_{k}$ a subset of criteria, and $c_{i} \in C_{k}$, then we apply the conjunctive rule of combination to obtain $m_{C_{k}}$ :

$$
m_{C_{k}}=\bigcirc_{j=1}^{h} m_{c_{i}}
$$

where $h$ is the number of items of a specific group of criteria $C_{k}$.

Finally, these obtained bba's will be discounted by their corresponding measure of reliability. We apply the same process used in Equations 19 and 20 to get $m_{C_{k}}^{\alpha_{k}}$.

\subsection{Decision making}

To this end, and after updating the priorities of the alternatives sets with respect to their set of criteria, we must combine the overall bba's in order to help the expert to make a decision:

$$
m_{\text {final }}=\bigcirc_{j=1}^{l} m_{C_{k}}^{\alpha_{k}}
$$

where $l$ is the number of subsets of criteria.

In this context, we choose to transform the final bba into pignistic probabilities using Equation 10. The decision maker will choose the alternative which has the highest value of pignistic probabilities. 


\section{$4 \quad$ PVC life cycle application}

The main aim of this section is to show an applicable way of our proposed approach. In fact, the potential of the belief AHP method may be illustrated by considering a real application problem. Here, we consider "the PVC (Polyvinyl chloride) life cycle" especially "the end of life phase". The problem considered attempts to rank countries based on their environmental impact to the disposal of PVC product. Thus, our challenge is to use or not the PVC in general, but to know in which country the environmental impact is less important for the destruction of a kilogram of PVC?

By following the Belief AHP procedure described in the previous section, the decision maker has to identify the set of criteria and alternatives and he has to indicate his preferences. Then, the following can be done manually or automatically by our implemented software.

\subsection{Identification of the candidate criteria and alternatives}

Following discussion with the knowledgeable expert, it was decided to restrict the number of criteria to four areas:

1. Acidification $\left(C_{1}\right)$ : the gradual decrease in the $\mathrm{pH}$ of the oceans.

2. Human toxicity infinite $\left(C_{2}\right)$ : this category concerns effects of toxic substances on the human environment.

3. Fresh water aquatic ecotoxicity infinite $\left(C_{3}\right)$ : this category indicator refers to the impact on fresh water ecosystems, as a result of emissions of toxic substances to air, water and soil.

4. Terrestrial ecotoxicity infinite $\left(C_{4}\right)$ : this category refers to impacts of toxic substances on terrestrial ecosystems.

Apart from the four criteria, the initial interview also identified six selected countries: "Switzerland (SW), France (FR), USA (US), England (ENG), Italy (I) and Spain (S)" which make the set of alternatives. We would generate a decision hierarchy by which it is possible to evaluate different alternatives. A figure showing the different levels of the hierarchy is presented (see Fig. 2).

\subsection{Computing the weight of considered criteria}

Given the necessary details of the criteria, the decision maker was asked to indicate his level of preference between them. Importantly, he was made 


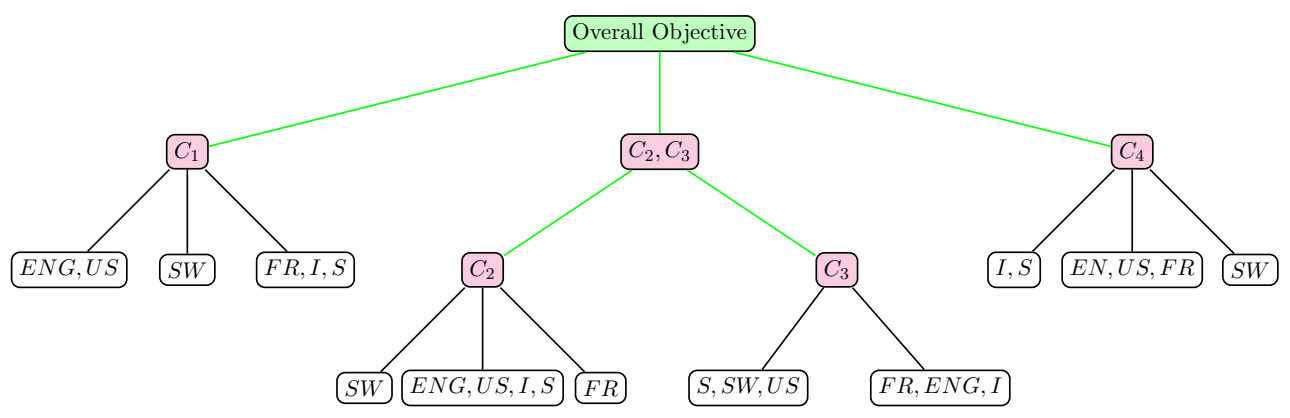

Figure 2: Belief AHP hierarchy.

aware that when assigning the same scale values to criteria, he was grouping them in the same subset.

To evaluate the responses of the pair-wise comparison question, the decision maker will be able to express his judgment with some degrees of uncertainty. That's, each element of the matrix will be labeled by a bba expressing a belief on judgment choice. So, the question is how to construct these bba's to obtain uncertain preferences. For collecting these expert beliefs, we have used a method for eliciting the expert opinions and we have generated associated belief functions (see Table 2).

Table 2: The weights preferences assigned to the criteria according to the expert's opinion

\begin{tabular}{|c|c|c|c|}
\hline & $\left\{C_{1}\right\}$ & $\left\{C_{4}\right\}$ & $\Omega_{1}=\left\{C_{2}, C_{3}\right\}$ \\
\hline$\left\{C_{1}\right\}$ & $m_{\left\{C_{1}\right\}}^{\Omega_{\left\{C_{1}\right\}}}\left(\Omega_{\left\{C_{1}\right\}}\right)=1$ & $\begin{array}{c}m_{\left\{C_{4}\right\}}^{\Omega_{\left\{C_{1}\right\}}}(\{\text { yes }\})=0.4 \\
\Omega_{\left\{C_{1}\right\}}\left(\Omega_{\left\{C_{1}\right\}}\right)=0.6 \\
m_{\left\{C_{4}\right\}}\end{array}$ & $\begin{array}{l}m_{\Omega_{1}}^{\Omega_{\left\{C_{1}\right\}}}(\{\text { yes }\})=0.9 \\
\Omega_{\left\{C_{1}\right\}}\left(\Omega_{\left\{C_{1}\right\}}\right)=0.1\end{array}$ \\
\hline$\left\{C_{4}\right\}$ & $\begin{array}{c}m_{\left\{C_{1}\right\}}^{\Omega_{\left\{C_{4}\right\}}}(\{n o\})=0.4 \\
m_{\left\{C_{4}\right\}}\left(\Omega_{\left\{C_{4}\right\}}\right)=0.6 \\
m_{\left\{C_{1}\right\}}\end{array}$ & $m_{\left\{C_{4}\right\}}^{\Omega_{\left\{C_{4}\right\}}}\left(\Omega_{\left\{C_{4}\right\}}\right)=1$ & $\begin{array}{l}m_{\Omega_{1}}^{\Omega_{\left\{C_{4}\right\}}}(\{n o\})=0.3 \\
m_{\left\{C_{4}\right\}}\left(\Omega_{\left\{C_{4}\right\}}\right)=0.7\end{array}$ \\
\hline$\Omega_{1}=\left\{C_{2}, C_{3}\right\}$ & $\begin{array}{l}m_{\left\{C_{1}\right\}}^{\left.\Omega_{1}\right\}}(\{n o\})=0.9 \\
m_{\Omega_{1}}\left(\Omega_{\Omega_{1}}\right)=0.1\end{array}$ & $\begin{array}{l}m_{\left\{C_{4}\right\}}^{\Omega_{\Omega_{1}}}(\{y e s\})=0.3 \\
m_{\left\{\Omega_{1}\right\}}^{\Omega_{\Omega_{4}}}\left(\Omega_{\Omega_{1}}\right)=0.7\end{array}$ & $m_{\Omega_{1}}^{\Omega_{\Omega_{1}}}\left(\Omega_{\Omega_{1}}\right)=1$ \\
\hline
\end{tabular}

From Table 2, we conclude that the decision maker has identified three subsets of criteria $\left\{C_{1}\right\},\left\{C_{4}\right\}$, and $\left\{C_{2}, C_{3}\right\}$. For instance, the expert may say that $\left\{C_{1}\right\}$ is evaluated to be more important than $\left\{C_{4}\right\}$ with a confidence degree of 0.4. That means, 0.4 of beliefs are exactly committed to the criterion $\left\{C_{1}\right\}$ is more important than $\left\{C_{4}\right\}$, whereas 0.6 is assigned to the whole frame of discernment (ignorance).

Then, the next step consists in combining the bba's corresponding to 
each criterion using the Equation 15. The obtained bba's are reported in Table 3.

Table 3: Belief pair-wise matrix: Partial combination

\begin{tabular}{|c|c|c|c|}
\hline & $C_{1}$ & $C_{4}$ & $\Omega_{1}=\left\{C_{2}, C_{3}\right\}$ \\
\hline \multirow{3}{*}{ Weight } & $m^{\Omega_{\left\{C_{1}\right\}}(\{\text { yes }\})=0.94}$ & $m^{\Omega_{\left\{C_{4}\right\}}}(\{$ no $\})=0.58$ & $m^{\Omega_{\Omega_{1}}}(\{$ yes $\})=0.03$ \\
& $m^{\Omega_{\left\{C_{1}\right\}}\left(\Omega_{\left\{C_{1}\right\}}\right)=0.06}$ & $m^{\Omega_{\left\{C_{4}\right\}}\left(\Omega_{\left\{C_{4}\right\}}\right)=0.42}$ & $m^{\Omega_{\Omega_{1}}}(\{$ no $\})=0.63$ \\
& & & $m^{\Omega_{\Omega_{1}}(\emptyset)=0.27}$ \\
& & & $m^{\Omega_{\Omega_{1}}\left(\Omega_{\Omega_{1}}\right)=0.07}$ \\
\hline
\end{tabular}

After that, the resulting bba's are transformed into pignistic probabilities (see Table 4).

Table 4: Belief pair-wise matrix: pignistic probabilities

\begin{tabular}{|c|c|c|c|}
\hline & $\left\{C_{1}\right\}$ & $\left\{C_{4}\right\}$ & $\Omega_{1}=\left\{C_{2}, C_{3}\right\}$ \\
\hline BetP & $\begin{array}{c}\operatorname{Bet}^{\Omega_{\left\{C_{1}\right\}}}(\{y e s\})=0.97 \\
\operatorname{Bet}^{\Omega_{\left\{C_{1}\right\}}}(\{\text { no }\})=0.03\end{array}$ & $\begin{array}{c}\operatorname{Bet}^{\Omega_{\left\{C_{4}\right\}}}(\{y e s\})=0.21 \\
\operatorname{Bet}^{\Omega_{\left\{C_{4}\right\}}(\{n o\})}=0.79\end{array}$ & 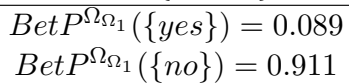 \\
\hline
\end{tabular}

\subsection{Computing the alternatives priorities}

Like the criterion level, the judgments between decision alternatives over different criteria are dealt within an identical manner. For example, to evaluate the alternatives according to the criterion $C_{1}$ (Acidification), the decision maker is asked to evaluate the following subsets of alternatives: $\{E N G, U S\},\{F R, I, S\}$ and $\{S W\}$, and we get the matrix presented in Table 5 .

Table 5: Belief pair-wise matrix regarding $C_{1}$ criterion

\begin{tabular}{|c|c|c|c|}
\hline$C_{1}$ & $\{E N G, U S\}$ & $\{F R, I, S\}$ & $\{S W\}$ \\
\hline$\{E N G, U S\}$ & $m_{\{E N G, U S\}}^{\Theta_{\{E N G, U S\}}}\left(\Theta_{\{E N G, U S\}}\right)=1$ & $\begin{array}{l}m_{\{F R, I, S\}}^{\Theta_{\{E N G, U S\}}}(\{n o\})=0.05 \\
m_{\{F N G, U S\}} \Theta_{\{F R, I, S\}}\left(\Theta_{\{E N G, U S\}}\right)= \\
0.95\end{array}$ & $\begin{array}{l}m_{\{S W\}}^{\Theta_{\{E N G, U S\}}}(\{y e s\})=0.4 \\
m_{\{E N G, U S\}}\left(\Theta_{\{E N G, U S\}}\right) \\
0.6\end{array}$ \\
\hline$\{F R, I, S\}$ & $\begin{array}{c}m_{\{E N G, U S\}}^{\Theta_{\{F R, I, S\}}}(\{y e s\})=0.05 \\
m_{\{E N, I, S\}}\left(\Theta_{\{F R, I S\}}\left(\Theta_{\{F R, I, S\}}\right)=0.95\right.\end{array}$ & $m_{\{F R, I, S\}}^{\Theta_{\{F R, I, S\}}}\left(\Theta_{\{F R, I, S\}}\right)=1$ & $\begin{array}{l}m_{\{S W\}}^{\Theta_{\{F R, I, S\}}}(\{\text { yes }\})=0.2 \\
m_{\{S W, I, S\}}\left(\Theta_{\{F R, I, S\}}\right)=0.8\end{array}$ \\
\hline$\{S W\}$ & $\begin{array}{c}m_{\{E N G, U S\}}^{\Theta_{\{S W\}}}(\{n o\})=0.4 \\
m_{\{E N G, U S\}}^{\Theta_{\{S W\}}}\left(\Theta_{\{S W\}}\right)=0.6\end{array}$ & $\begin{array}{l}m_{\{F R, I, S\}}^{\Theta_{\{S W\}}}(\{n o\})=0.2 \\
m_{\{F R, I, S\}}\left(\Theta_{\{S W\}}\right)=0.8\end{array}$ & $m_{\{S W\}}^{\Theta_{\{S W\}}}\left(\Theta_{\{S W\}}\right)=1$ \\
\hline
\end{tabular}

As in the criterion level, for the subset of alternatives $\{E N G, U S\}$, we use the Equation 15 in order to combine the obtained bba (see Table 6):

$$
m^{\Theta_{\{E N G, U S\}}}=m_{\{E N G, U S\}}^{\Theta_{\{E N G, U S\}}} @ m_{\{F R, I, S\}}^{\Theta_{\{E N G, U S\}}} @ m_{\{S W\}}^{\Theta_{\{E N G, U S\}}}
$$


Then, a similar process is repeated for the rest of alternatives, and we get $m^{\Theta_{\{F R, I, S\}}}$ and $m^{\Theta_{\{S W\}}}$.

Table 6: Belief pair-wise matrix: Partial combination

\begin{tabular}{|c|c|c|c|}
\hline$C_{1}$ & $\{E N G, U S\}$ & $\{F R, I, S\}$ & $\{S W\}$ \\
\hline \multirow{4}{*}{ Priority } & $m^{\Theta_{\{E N G, U S\}}}(\{y e s\})=0.38$ & $m^{\Theta_{\{F R, I, S\}}(\{y e s\})=0.24}$ & $m^{\Theta_{\{S W\}}}(\{n o\})=0.52$ \\
& $m^{\Theta_{\{E N G, U S\}}(\{n o\})=0.03}$ & $m^{\Theta_{\{F R, I, S\}}\left(\Theta_{\{F R, I, S\}}\right)=0.76}$ & $m^{\Theta_{\{S W\}}\left(\Theta_{\{S W\}}\right)=0.48}$ \\
& $m^{\Theta_{\{E N G, U S\}}(\emptyset)=0.02}$ & & \\
\hline
\end{tabular}

Subsequently, we proceed now with the standardization of our frame of discernment. By applying the Equation 16, in the subset of alternatives $\{E N G, U S\}$ for example, we get the following bba:

$$
\begin{gathered}
m^{\Theta_{\{E N G, U S\}} \uparrow \Theta}(\{E N G, U S\})=m^{\Theta_{\{E N G, U S\}}}(\{y e s\}) \\
m^{\Theta_{\{E N G, U S\}} \uparrow \Theta}(\overline{\{E N G, U S\}})=m^{\Theta_{\{E N G, U S\}}}(\{n o\}) \\
m^{\Theta_{\{E N G, U S\}} \uparrow \Theta}(\Theta)=m^{\Theta_{\{E N G, U S\}}}\left(\Theta_{\{E N G, U S\}}\right)
\end{gathered}
$$

To simplify, we can note by $m_{\{E N G, U S\}}^{\Theta}$ the bba $m_{\{E N G, U S\}}^{\Theta^{\uparrow}}$, and the similar process is repeated for the rest of alternatives. The obtained matrix is presented in Table 7 .

Table 7: Belief pair-wise matrix: Refinement

\begin{tabular}{|c|c|c|c|}
\hline$C_{1}$ & $\{E N G, U S\}$ & $\{F R, I, S\}$ & $\{S W\}$ \\
\hline \multirow{4}{*}{ Priority } & $m_{\{E N G, U S\}}^{\Theta}(\{E N G, U S\})=0.38$ & $m_{\{F R, I, S\}}^{\Theta}(\{F R, I, S\})=0.24$ & $m_{\{S W\}}^{\Theta}(\{F R, I, S, U S, E N G\})=0.52$ \\
& $m_{\{E N G, U S\}}^{\Theta}\{(F R, S W, I, S\})=0.03$ & $m_{\{F R, I, S\}}^{\Theta}(\Theta)=0.76$ & $m_{\{S W\}}^{\Theta}(\Theta)=0.48$ \\
& $m_{\{E N G, U S\}}^{\Theta}(\emptyset)=0.02$ & & \\
& $m_{\{E N G, U S\}}^{\Theta}(\Theta)=0.57$ & & \\
\hline
\end{tabular}

Finally, the obtained bba's can be directly combined using the conjunctive rule of combination (see Table 8).

Table 8: The bba $m_{C_{1}}$

\begin{tabular}{|c|c|c|c|c|c|c|}
\hline & $\emptyset$ & $\{F R, I, S\}$ & $\{F R, I, S, E N G, U S\}$ & $\{F R, S W, I, S\}$ & $\{E N G, U S\}$ & $\Theta$ \\
\hline$m_{C_{1}}$ & 0.1113 & 0.1559 & 0.2252 & 0.0109 & 0.2887 & 0.2079 \\
\hline
\end{tabular}

Then, as shown in the previous step, the computation procedure is repeated for the rest of comparison matrices to get Table 9 . 
Table 9: The bba's $m_{C_{2}}, m_{C_{3}}$ and $m_{C_{4}}$

\begin{tabular}{|l|c|c|c|c|c|c|}
\hline & $\emptyset$ & $\{F R\}$ & $\{S W\}$ & $\{E N G, U S, I, S\}$ & $\Theta$ & \\
\hline$m_{C_{2}}$ & 0.541 & 0.128 & 0.012 & 0.226 & 0.093 & \\
\hline \hline & $\{S, S W, U S\}$ & $\Theta$ & & & & \\
\hline$m_{C_{3}}$ & 0.84 & 0.16 & & & & \\
\hline \hline & $\emptyset$ & $\{E N G, U S, F R\}$ & $\{I, S\}$ & $\{S W\}$ & $\{S W, I, S\}$ & $\Theta$ \\
\hline$m_{C_{4}}$ & 0.261 & 0.26 & 0.17 & 0.009 & 0.203 & 0.097 \\
\hline
\end{tabular}

\subsection{Updating the alternatives' priorities}

After computing the belief functions for each set of alternatives with respect to each criterion, we must update this bba with their corresponding measure of reliability.

Firstly, this step concerns the groups of criteria, that is the subset $\left\{C_{2}, C_{3}\right\}$. Therefore, by using the Equation 21, we propose to combine the bba relative to the $C_{2}$ and $C_{3}$ criteria $\left(m_{C_{2}, C_{3}}=m_{C_{2}} @ m_{C_{3}}\right)$.

Then, these obtained bba's are discounted by the measure of reliability $\beta_{C_{2}, C_{3}}=0.089$ (obtained from Table 4 and using the Equation 18). So, we use Equations 19 and 20 to get the Table 10.

Table 10: The bba $m_{C_{2}, C_{3}}$ and $m_{C_{2}, C_{3}}^{\alpha_{C_{2}, C_{3}}}$

\begin{tabular}{|c|c|c|c|c|c|c|c|}
\hline & $\emptyset$ & $\{F R\}$ & $\{S W\}$ & $\{U S, S\}$ & $\{E N G, U S, I, S\}$ & $\{S, S W, U S\}$ & $\Theta$ \\
\hline$m_{C_{2}, C_{3}}$ & 0.6488 & 0.0204 & 0.012 & 0.1898 & 0.0361 & 0.0781 & 0.0148 \\
\hline$m_{C_{2}, C_{3}}^{\alpha_{C}, C_{3}}$ & 0.0577 & 0.0018 & 0.001 & 0.0168 & 0.0032 & 0.0069 & 0.9126 \\
\hline
\end{tabular}

After that, the next step concerns the criterion $\left\{C_{1}\right\}$ and $\left\{C_{4}\right\}$. The relative bba are directly discounting using Equations 19 and 20, where the reliability measures $\beta_{C_{1}}=0.97$ and $\beta_{C_{4}}=0.21$ (see Table 11).

Table 11: The bba $m_{C_{1}}^{\alpha_{C_{1}}}$ and $m_{C_{4}}^{\alpha_{C_{4}}}$ after discounting

\begin{tabular}{|l|c|c|c|c|c|c|}
\hline & $\emptyset$ & $\{F R, I, S\}$ & $\{F R, I, S, E N G, U S\}$ & $\{F R, S W, I, S\}$ & $\{E N G, U S\}$ & $\Theta$ \\
\hline$m_{C_{1}}^{\alpha_{C_{1}}}$ & 0.1080 & 0.1512 & 0.2184 & 0.0105 & 0.28 & 0.2319 \\
\hline \hline$\omega_{C_{4}}^{\alpha_{C_{4}}}$ & 0.0548 & $\{E N G, U S, F R\}$ & $\{I, S\}$ & $\{S W\}$ & $\{S W, I, S\}$ & $\Theta$ \\
\hline
\end{tabular}

\subsection{Synthetic utility and decision making}

The final step is then to choose the best alternatives. First, we apply the conjunctive rule of combination (Equation 22), that leads us to get a single 
bba denoted by $m_{\text {final }}$. Then, we transform the obtained bba to a pignistic probability and we get this final result (see Table 12).

Table 12: The final result using the belief AHP approach

\begin{tabular}{|c|c|c|c|c|c|c|}
\hline Alternatives & $E N G$ & $U S$ & $S$ & $F R$ & $I$ & $S W$ \\
\hline BetP & 0.2328 & 0.2464 & 0.1676 & 0.1512 & 0.1569 & 0.0451 \\
\hline
\end{tabular}

The decision maker wants to know in which country the environmental impact is less important for the destruction of a kilogram of PVC.

The alternatives are now ranked according to their pignistic probabilities, as follows: $\{U S\},\{E N G\},\{S\},\{I\},\{F R\}$ and $\{S W\}$. Consequently, "USA" is the recommended country since it has the highest values.

For the sake of comparison, we have assumed that the expert is able to express his preferences with certainty and we have used the standard AHP method. We have obtained the following ranking: $\{F R\},\{I\},\{E N G\},\{S\}$, $\{U S\}$ and $\{S W\}$. We can conclude that, when the expert freely expresses his preferences the obtained result may be affected. Thus, we can obtain a more accurate decision since we have tried to closely model the decision maker assessment.

This research proposes that the differences between classic AHP method and belief AHP approach are raised by two potential factors. One, belief AHP method can provide the decision maker to express his preferences with some uncertainty and imprecision rather than deterministic value options. This gives an advantage over classical AHP method in solving complex problems. Besides, by using our approach, we try to model all the elicitated information. Indeed, our method expects to find the most cautious solution. Second, by using belief AHP method the number of comparisons is reduced. So, the use of subsets of criteria and/or alternatives for pair-wise comparison is hence the second factor.

In our case for example, if we have adopted the classical AHP, then there would be 15 comparisons per criterion between the decision alternatives level. That means, we will get 60 comparisons, and at the criterion level, we will have 6 comparisons. As a result, the number of pair-wise comparisons is then 66. However, by using the belief AHP, the number of comparisons decreases because instead of using single elements, we have used subsets. In fact, in our case the decision maker had made 3 pair-wise comparisons at the criterion level, and at the alternative level, he had made 10 pair-wise comparisons. In the worst case, the number of pair-wise comparisons is equal to the standard one, when singleton criterion is compared to singleton 
alternative.

\section{Experimental study}

In this Section, the performances of the proposed method, belief AHP, are compared. Firstly, the evaluation algorithm used in the comparisons is described. Secondly, the method is tested on randomly generated matrices. Then, we discuss the obtained results.

\subsection{Evaluation algorithm}

Despite the possible differences between the obtained results of two MCDM methods, we cannot conclude the superiority of one over another. Unless we have a solid basis, we compare the ranking results against the closeness of the rankings of each method to the actual rankings.

To do so, we need to compare each set of rankings provided by AHP and the proposed method with a ranking that has already been produced by an alternative, yet reliable ranking method. This alternative ranking will be considered as a basis, or actual ranking of the alternatives and will be used to measure the closeness of the rankings provided by AHP, belief AHP to reality. These evaluations are provided in the following Sections.

A method which is accurate in MCDM problems should also be accurate in single dimensional problems. Therefore, we use the Weighted Sum Model (WSM) method since in single-dimensional environment, [41] it yields the most reasonable results. Hence, some previous works compare the obtained results using WSM to those obtained by other MCDM method. [38] [39] This evaluation criterion has been applied in order to evaluate crisp and fuzzy MCDM methods. [38] [39] We therefore consider that at this level, there is no need to compare our method to other multi-criteria decision making approaches since we compare our solution to one of the most reliable method in a unidimensional context.

Besides, WSM is the simplest and still the widest used MCDM method. In this method, each criterion is given a weight, and the sum of all weights must be 1. Each alternative is assessed with regard to every criterion. The overall or composite performance score of an alternative is given by the equation:

$$
P_{i}=\sum_{j} v_{i j} * \omega_{j}
$$

where $P_{i}$ is the priority of each alternative, $\omega_{j}$ is the weight of each criterion and $v_{i j}$ is the score of each alternative regarding each criterion. 
In order to overcome these issues, in the next Section, we test a simulation algorithm that compares the ranking results of belief AHP under different scenarios.

\subsection{Simulation algorithm}

To generate reliable data for a numerical analysis in AHP, simulation has been extensively used in prior research. [39] [38] The simulation algorithm is summarized as follows:

1. We generate a random matrix for the decision performance and another one to represent the weight of each decision criteria. Based on these two matrices, the overall scores and ranks of the decision alternatives are calculated. These steps are usual steps in the WSM method.

2. From the performance matrix, we generated pair-wise comparison matrices of different alternatives that are compared to each criterion.

3. Each pair-wise matrix is transformed into belief pair-wise comparison. Indeed, the resulting bbas has only one focal element since we are in a certain context:

- If the actual preference value regarding the alternative $a$ with bbm, $m(\{y e s\})=1$ (If $1<p \leq 9)$.

- If $p=1$ then $m(\Theta)=1$

- If $\frac{1}{9} \leq p \leq \frac{1}{2}$ then $m(\{n o\})=1$

4. We apply the suggested method to compute the overall priorities and to rank alternatives.

5. We compare the obtained result with the ranking of the WSM method.

In order to gain a deeper understanding, a computational study was undertaken. The data were random numbers from the interval [1,9] (in order to be compatible with the numerical properties of the Saaty scale). In these test problems, the number of alternatives was equal to the following values: $3,4,5,6,7,8,9$, and 10 . Similarly, the number of criteria was equal to $3,4,5,6,7,8,9$, and 10. Psychological experiments have shown that individuals cannot simultaneously compare more than seven objects (plus or minus two). [40] Therefore, we choose that the number of criteria and alternatives in the analysis should not exceed 10 . Thus, a total of $(8 \times 8)$ different cases 
were examined with 100 replications (in order to derive statistically significant results) per each case. Each random problem was solved using the original and belief AHP methods.

\subsection{Simulation results}

One of the difficulties in comparing many MCDM methods is that there is rarely any way to check the accuracy of the methods. At the same time, it must be understood that these methods have different relative advantages in dealing with different types of data such as ordinal and uncertain data. The intent is not to establish the dominance of any one method, but rather to compare the relative accuracy in the case of known outcome.

Table 13: Percentage of contradiction (\%) between the WSM and the AHP

\begin{tabular}{ccccccccc}
\hline & \multicolumn{8}{c}{ Number of alternatives } \\
Number of criteria & 3 & 4 & 5 & 6 & 7 & 8 & 9 & 10 \\
\hline 3 & 7 & 7 & 9 & 9 & 10 & 11 & 13 & 12 \\
4 & 7 & 7 & 9 & 9 & 11 & 11 & 11 & 11 \\
5 & 7 & 7 & 9 & 9 & 12 & 11 & 11 & 11 \\
6 & 8 & 6 & 9 & 10 & 11 & 12 & 13 & 13 \\
7 & 8 & 6 & 10 & 10 & 11 & 12 & 13 & 13 \\
8 & 8 & 6 & 10 & 10 & 10 & 1 & 12 & 13 \\
9 & 8 & 6 & 10 & 10 & 10 & 12 & 12 & 14 \\
10 & 9 & 6 & 11 & 11 & 10 & 12 & 14 & 14 \\
\hline
\end{tabular}

Table 14: Percentage of contradiction (\%) between the WSM and the belief AHP

\begin{tabular}{ccccccccc}
\hline & \multicolumn{10}{c}{ Number of alternatives } \\
Number of criteria & 3 & 4 & 5 & 6 & 7 & 8 & 9 & 10 \\
\hline 3 & 5 & 5 & 5 & 6 & 9 & 9 & 9 & 10 \\
4 & 5 & 5 & 5 & 6 & 9 & 9 & 9 & 11 \\
5 & 7 & 7 & 7 & 6 & 10 & 9 & 10 & 11 \\
6 & 7 & 7 & 9 & 6 & 10 & 9 & 10 & 12 \\
7 & 8 & 8 & 9 & 6 & 10 & 10 & 10 & 12 \\
8 & 8 & 8 & 9 & 10 & 10 & 10 & 10 & 12 \\
9 & 8 & 8 & 10 & 10 & 10 & 10 & 10 & 12 \\
10 & 8 & 8 & 10 & 10 & 10 & 10 & 10 & 12 \\
\hline
\end{tabular}


We also notice, from Tables 13 and 14, that there is a significant difference in the percentage of contradiction between AHP and belief AHP methods. Most importantly, when comparing the overall results, we can remark that problems with less alternatives yielded fewer ranking contradictions. As it can be seen, the number of criteria did not play a prime role. For instance, from Table 14, our method is compared regarding 5 criteria and different alternatives the percentage of contradiction in almost cases is under $10 \%$.

Importantly, belief AHP method uses only the minimum information to model the decision maker preferences. However, more uncertainty can be handled within the scale of preferences.

\section{Conclusion}

In this paper, a new approach inspired of the AHP method was developed. Our proposed method, named belief AHP, is based on the belief function framework. Indeed, this approach is shown to model the uncertain decision maker's preferences and to reduce the number of pair-wise comparisons. Instead of comparing single alternatives regarding single criterion, our belief AHP compares the sets of decision alternatives with respect to certain groups of criteria. Moreover, our method has adopted a yes/no choice and for each response we assign a degree of belief. Consequently, our approach follows the same outline as AHP method in uncertain environment. Finally, to illustrate the feasibility of our approaches and to judge their performances, we have applied our proposed methods on a real application problem. Our aim was to choose the appropriate country, where its environmental impact is the least important for the destruction of a kilogram of PVC, on the basis of several criteria.

Nevertheless, the proposed work is still subject to improvements. It can be extended into different directions. In fact, our method can be improved by defining a new consistency ratio in a belief function framework. Thus,

the proposed method will be more flexible, if it will be able to calculate the consistency of a belief pair-wise matrix. Further work may be suggested on the comparison between the belief AHP approach and other methods.

\section{References}

[1] T. Saaty, How to make a decision: The Analytic Hierarchy Process, European Journal of Operational Research. 481990 9-26. 
[2] P. Joaquin, Some Comments on the Analytic Hierarchy Process, The Journal of the Operational Research Society. 41(6) $19901073-1076$.

[3] R. D. Holder, Some Comments on Saaty's AHP, Management Science. 41 1995 1091-1095.

[4] G. Shafer, A Mathematical Theory of Evidence Princeton University Press, 1976.

[5] Ph. Smets, The Application of the Transferable Belief Model to Diagnostic Problems. International Journal of Intelligent Systems 131998 $127-158$.

[6] A. Ennaceur, Z. Elouedi and E. Lefevre, Handling Partial Preferences in the Belief AHP Method: Application to Life Cycle Assessment. Proceedings of the International Conference of the Italian Association for Artificial Intelligence, LNAI 6934, Springer-Verlag 2011 396-401.

[7] T. J. Stewart, A critical survey on the status of multiple criteria decision making theory and practice. OMEGA 201992 569-586.

[8] Ch. Huanga, P. Chub and Y. Chiang, A fuzzy AHP application in government sponsored R-D project selection. OMEGA 36(6) 20081038 1052.

[9] M. Zeleny, Multiple Criteria Decision Making McGraw-Hill Book Company, 1982.

[10] T. Saaty, A scaling method for priorities in hierarchical structures. Journal of Mathematical Psychology 151977 234-281.

[11] T. Saaty, The Analytic Hierarchy Process. McGraw-Hill, New-York, 1980.

[12] T. Saaty, An exposition of the AHP in reply to the paper "Remarks on the Analytic Hierarchy Process". Management Science 36(3) 1990 $426-447$.

[13] P.J.M. Van Laarhoven and W. Pedrycz, A fuzzy extension of Saaty's priority theory. Fuzzy Sets and Systems 111983 199-227.

[14] F. A. Lootsma, Fuzzy Logic for planning and Decision-Making Kluwer Academic Publishers, 1997. 
[15] I. Basak, Probabilistic judgments specified partially in the Analytic Hierarchy Process. European Journal of Operational Research 1081998 153-164.

[16] B. Schoner and W. C. Wedley, Ambiguous criteria weights in AHP: consequences and solutions. Decision Sciences $201989462-475$.

[17] M. Beynon, B. Curry and P. Morgan, The Dempster-Shafer theory of evidence: An alternative approach to multicriteria decision modelling. OMEGA 2000 28(1) 37-50.

[18] M. Beynon, DS/AHP method: A mathematical analysis, including an understanding of uncertainty. European Journal of Operational Research 1402002 148-164.

[19] J. Dezert, J. M. Tacnet, M. Batton-Hubert and F. Smarandache, MultiCriteria Decision Making based on DSmT-AHP. Workshop on the Theory of Belief Functions, 2010.

[20] F. Smarandache and J. Dezert, Advances and Applications of DSmT for Information Fusion Collected works American Research Press, 2004.

[21] Ph. Smets, The combination of evidence in the Transferable Belief Model. IEEE Pattern analysis and Machine Intelligence 121990447 458.

[22] Ph. Smets and R. Kennes, The Transferable Belief Model. Artificial Intelligence 661994 191-234.

[23] G. Shafer, The combination of evidence. International Journal of Intelligent Systems 11986 155-180.

[24] Ph. Smets, Transferable Belief Model for expert judgments and reliability problems. Reliability Engineering and System Safety 381992 59-66.

[25] D. Dubois and H. Prade, A set-theoretic view of belief functions: logical operations and approximations by fuzzy sets. International Journal of General Systems 12(3) 1986 193-226.

[26] L. V. Utkin, A new ranking procedure by incomplete pair-wise comparisons using preference subsets. Intelligent Data Analysis 13(2) 2009 229-241. 
[27] M. Beynon, An analysis of distributions of priority values from alternative comparison scales within AHP. European Journal of Operational Research 1402002 105-118.

[28] M. Beynon, A method of aggregation in DS/AHP for group decisionmaking with the non-equivalent importance of individuals in the group. Computers and Operations Research 32(7) 2005 1881-1896.

[29] L. V. Utkin and N. Simanova, Multi-criteria decision making by incomplete preferences. Journal of Uncertain Systems 22008 255-266.

[30] V.M. Rao Tummala and H. Ling, A Note on the Computation of the Mean Random Consistency Index of the Analytic Hierarchy Process AHP. Theory and Decision 441998 221-230.

[31] Y. M. Wanga and T. M.S. Elhag, An approach to avoiding rank reversal in AHP. Decision Support Systems 422006 1474-1480.

[32] J. Benítez, X. Delgado-Galván, J. Izquierdo and R. Pérez-García, An approach to AHP decision in a dynamic context. Decision Support Systems 53(3) 2012 499-506.

[33] L. V. Utkin and N. Simanova, The DS/AHP method under partial information about criteria and alternatives by several levels of criteria. International Journal of Information Technology and Decision Making $2012307-326$.

[34] C.N. Liao, A fuzzy approach to business travel airline selection using an integrated AHP-TOPSIS-MSGP methodology. International Journal of Information Technology and Decision Making 122013 241-258.

[35] Z. Turskis, E. K. Zavadskas and V. Kutut, A model based on Aras-G and AHP methods for multiple criteria prioritizing of heritage value. International Journal of Information Technology and Decision Making 122013 45-73.

[36] A. Mayyas, Q. Shen, A. Mayyas, D. Shan, A. Qattawi and M. Omar, Using quality function deployment and analytical hierarchy process for material selection of body-in-white. Materials and Design 322011 27712782 .

[37] A. Qattawi, A. Mayyas, M. Abdelhamid and M. Omar, Incorporating quality function deployment and analytical hierarchy process in a 
knowledge-based system for automotive production line design. International Journal of Computer Integrated Manufacturing 262013 839-856.

[38] E. Triantaphyllou and S. H. Mann, An Examination of the Effectiveness of Multi-Dimensional Decision-Making Methods: A Decision-Making Paradox. Decision Support Systems 5(3) 1989 303-312.

[39] E. Triantaphyllou and C. T. Lin, Development and Evaluation of Five Fuzzy Multiattribute Decision-Making Methods. International Journal of Approximate Reasoning 14(4) 1996 281-310.

[40] G. A. Miller, The Magical Number Seven Plus or Minus Two: Some Limits on Our Capacity for Processing Information. Psychological Review 63 1956 81-97.

[41] P. C. Fishburn, Additive Utilities with Incomplete Product Set: Applications to Priorities and Assignment. Operations Research 1967.

[42] Gang Kou and Changsheng Lin, A cosine maximization method for the priority vector derivation in AHP. European Journal of Operational Research 2352014 225-232.

[43] Gang Kou, Daji Ergu and Jennifer Shang, Enhancing Data Consistency in Decision Matrix: Adapting Hadamard Model to Mitigate Judgment Contradiction. European Journal of Operational Research 236 (1) 2014 261-271. 